\title{
La recherche biomédicale à la Faculté de Médecine de l'Université de Liège
}

\author{
Jacques Boniver
}

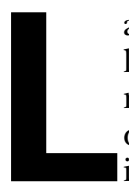
a Faculté de Médecine de l'Université de Liège a le même âge que l'Université dont elle est une des plus importantes facultés: il y a 5 ans, elle fêtait son $175^{\mathrm{e}}$ anniversaire. L'Université de Liège, d'abord dépendante de l'État belge, est devenue une Université de la Communauté française de Belgique, à la suite des réformes institutionnelles de ces dernières années.

\section{La Faculté de Médecine et I'Hôpital universitaire}

La Faculté de Médecine a trouvé son berceau naturel dans un hôpital de Liège, l'hôpital de Bavière, créé en 1603.

Lorsque le Recteur M. Dubuisson décida de l'implantation de l'Université sur le site du Sart Tilman, à $12 \mathrm{~km}$ environ du centre-ville, s'engagea un débat passionné sur le devenir de l'hôpital de Bavière. Celui-ci, désuet, ne pouvait plus répondre aux besoins hospitaliers de la région liégeoise, ni en aucune façon accueillir les développements technologiques de la médecine moderne. Par ailleurs, il fonctionnait sur un mode hybride, les activités liées à l'hospitalisation étant gérées par la Ville de Liège, à travers son service d'Assistance publique, les activités strictement médicales étant gérées par l'Université.

La Ville de Liège décida de créer son propre hôpital sur le site de la Citadelle, sur la rive gauche de la Meuse tandis que l'Université entamait la construction du «Centre Hospitalier Universitaire ", au Sart Tilman sur la rive droite. Ce projet subit de nombreuses vicissitudes, d'une part, en raison de l'insuffisance des crédits alloués par l'État et, d'autre part, en raison de changements importants dans la politique hospitalière. En effet, au début des années 1980, le pays comptait trop de lits d'hôpitaux et, sur la place de Liège, les projets de construction de l'Hôpital de la Citadelle et de l'hôpital universitaire, auxquels il fallait ajouter des projets de développement d'hôpitaux privés, entraînaient un accroissement considérable du nombre de lits. Dès lors, chaque institution fut obligée de limiter ses prétentions.

L'Hôpital universitaire au Sart Tilman ne put accueillir que 639 lits, au lieu des 1100 prévus initialement, ce qui était insuffisant pour accueillir tous les services de stages pour les étudiants de $2^{\mathrm{e}}$ et $3^{\mathrm{e}}$ cycles. Précisons que l'Université de Liège diplôme annuellement environ 120 docteurs en médecine dont environ la moitié poursuit des études de spécialisation. Pour former un tel nombre d'étudiants, l'Université disposait en théorie, de 935 lits dits "académiques» pour l'enseignement clinique. Aussi, environ 300 lits "académiques" furent installés à l'hôpital de la Citadelle. Il s'agit de la Pédiatrie universitaire, d'une grande partie de la Gynécologie-Obstétrique et de quelques autres services.

Cette implantation de lits académiques dans un hôpital non universitaire fut le premier chaînon d'un réseau rassemblant des hôpitaux avec lesquels l'Hôpital universitaire et l'Université établirent des conventions de collaboration structurée et approfondie. L'Université y installa quelques lits académiques. Dans le même temps, l'Hôpital universitaire devenait une entité juridique clairement séparée de l'Université, mais dépendante, comme elle, de la Communauté française de Belgique.

Ce réseau hospitalier trouve son importance d'une part dans la nécessaire hiérarchisation des soins et d'autre part dans les besoins de l'institution pour la formation des médecins. On ne peut concevoir un bon enseignement de la médecine sans une activité clinique intensive: c'est ce que ce réseau offre à nos étudiants ainsi qu'à ceux qui choisissent une formation de $3^{\text {e }}$ cycle les préparant à une spécialisation médicale.

\section{Le numerus clausus pour l'accès à la profession médicale}

L'enseignement de la Médecine en Belgique entre dans une grande période de mutation. La Belgique était le dernier pays de l'Union européenne à laisser tout à fait libre l'accès aux études de médecine, puis l'accès à la profession, dans le cadre de la Sécurité sociale. Conséquence: la Belgique est le pays où la densité médicale est la plus grande. Conséquence complémentaire: un certain nombre de médecins, généralistes ou spécialistes, ont une activité faible. Par ailleurs, le coût des soins de santé 
financés par la Sécurité sociale ne fait que croître.

Après plus de 20 ans de débat, le gouvernement belge vient de décider l'instauration d'un numerus clausus d'accès à la profession dans le cadre de la Sécurité sociale. La réduction est redoutable: d'ici 2004, le nombre de nouveaux médecins bénéficiant d'un tel accès sera réduit de moitié dans la partie francophone du pays. La Communauté Française, dont dépendent les Universités, a décidé de sélectionner à la fin du premier cycle*, ceux qui, 4 ans plus tard, auront accès à la profession. Pour réaliser cette sélection, les Universités ont modifié les programmes du premier cycle d'une part pour évaluer l'aptitude de l'étudiant à exercer la médecine en vue de la sélection, et d'autre part pour favoriser la réorientation des étudiants vers d'autres disciplines (pharmacie, sciences biomédicales, sciences de la Santé publique...). Tout cela a créé et créera encore beaucoup de remous.

Pour terminer la présentation de la Faculté, ajoutons qu'outre la section Médecine, elle comprend également l'École de Dentisterie - elle aussi soumise à un numerus clausus -, l'Institut de Pharmacie et l'Institut d'Éducation physique-Kinésithérapie.

\section{La recherche biomédicale}

Les laboratoires de recherche de la Faculté de Médecine sont pour la plupart rassemblés dans les deux «Tours de Pathologie " du complexe du Centre Hospitalier Universitaire au Sart Tilman. Certains laboratoires des sciences fondamentales (physiologie, biochimie, histologie) se trouvent encore au centre-ville, dans les Instituts Léon Fredericq et Auguste Swaen. Les chercheurs disposent, au Sart Tilman, d'une bibliothèque exceptionnelle.

\section{La cancérologie}

C'est certainement dans les domaines de la cancérologie, des neurosciences

\footnotetext{
* Pour rappel, en Belgique, le premier cycle des études de médecine comprend trois années de candidatures au cours desquelles sont enseignées les sciences de base, incluant l'homme normal et l'homme malade, puis un deuxième cycle de 4 docto-
}

et de l'endocrinologie que les chercheurs liégeois ont le plus rayonné, et rayonnent encore.

Les thèmes de recherche fondamentale, en cancérologie, vont des études sur le métabolisme des cancérogènes chimiques (P. Kremers), les protooncogènes (R. Winkler), l'angiogenèse et l'invasion tumorale, les défenses immunitaires antitumorales, l'apoptose jusqu'à la mise au point de nouveaux médicaments ou de traitements biologiques nouveaux, à l'hôpital.

De nombreux travaux sont centrés sur le rôle de l'environnement tissulaire dans le développement et la progression des cancers. D'autres recherches visent à mieux comprendre les mécanismes de défense de l'individu contre les tumeurs. Citons les travaux de G. De Giovanni qui, en collaboration avec le groupe de T. Boon, de l'Institut Ludwig de Bruxelles, étudie les réactions immunitaires cellulaires dirigées contre les mélanomes. D'autre part, Ph. Delvenne, M. Moutschen et leurs collaborateurs s'intéressent aux mécanismes d'immunotolérance dans les cancers du col utérin induits pars les papillomavirus humains et à leur rôle dans la progression des lésions. La recherche est centrée sur la mise en évidence d'une immunodéviation TH1/TH2 et sur des manipulations qui visent à la contrecarrer.

Parmi les recherches sur les cellules cancéreuses, il faut aussi citer celles qui visent à étudier le rôle potentiel de NF-kB, un facteur de transcription qu'un de nos chercheurs, V.Bours, a été parmi les premiers à décrire. Ce facteur est aussi étudié dans le stress oxydatif et dans des cellules infectées par le HIV-1 (voir l'article de V. Bours et al., p. 566 de ce numéro).

Insistons sur le fait que l'Hôpital universitaire servant de centre de référence en cancérologie clinique dans la région, une unité de greffe de moelle et de cellules souches hématopoḯtiques est particulièrement active; les recherches de G. Fillet, Y.Beguin et J.M. Paulus sur les cellules souches de la moelle, l'érythropoïèse et les récepteurs à la transferrine et sur les mégacaryocytes sont bien connus; le centre de radiothérapie est en constant développement et une unité de recherche en thérapie génique s'est constituée, se donnant comme priorité la thérapie génique des cancers du côlon.

\section{Les neurosciences}

Les neurosciences fondamentales et cliniques dont les promoteurs principaux sont G. Franck, A. Stevenaert et P. Delwaide sur le plan clinique et G. Moonen, T. Grisar et J. Schoenen sur le plan fondamental, sont un autre domaine de recherches très fructueux. Depuis les études in vitro sur des cellules gliales ou sur des cellules nerveuses (pour l'identification de diverses substances biologiques neurotropes ou neurotoxiques), jusqu'aux recherches menées par E. Salmon et P. Maquet en tomographie à positons visant à déceler la réactivité cérébrale lors du sommeil ou des exercices de mémorisation, on passe par des travaux importants sur l'épilepsie, les migraines, les démences, la paraplégie, les surdités,... (voir l'article de G. Moonen et al., p. 588 de ce numéro).

\section{L'endocrinologie}

L'endocrinologie, comme modèle de communications intercellulaires, est aussi un des fers de lance de nos recherches. Rappelons que cette discipline a trouvé son essor, à Liège, grâce au dynamisme de feu Paul Franchimont, qui avait trouvé, dans la radio-immunologie, l'outil nécessaire de dosage de multiples hormones.

Aujourd'hui, les recherches endocrinologiques sont menées chez J.J. Legros et V. Geenen, qui s'intéressent aux relations complexes entre le système nerveux central et le système hormonal hypophysaire et périphérique; V. Geenen étudie les liens éventuels entre ces systèmes et le système immunitaire. Ses études sur le thymus, où il a décelé la présence de plusieurs neuropeptides, l'ont amené à proposer des hypothèses originales quant aux mécanismes naturels de la tolérance, et de sa rupture, aboutissant à certaines maladies autoimmunes, comme le diabète.

Le groupe de G. Hennen poursuit ses travaux sur les interactions hormonales impliquées dans le contrôle des gonades et de l'appareil génital; 
citons en particulier des recherches sur le rôle des androgènes au niveau prostatique, ainsi que sur l'hormone de croissance placentaire, qui semble présenter des propriétés biologiques tout à fait passionnantes (voir article de G. Hennen et al., p. 572 de ce numéro).

La recherche en diabétologie est plutôt clinique. Menée par P. Lefèbvre et ses collaborateurs (A. Scheen, N. Paquot $e t a l$.), elle se concentre sur certains aspects liés au diabète de type 2, par la caractérisation de la boucle «glucose-insuline» et par les mécanismes de l'hyperglycémie. Cette équipe s'intéresse aussi aux mécanismes des complications du diabète. L'ostéoporose doit sa pathogénie à des déséquilibres hormonaux et l'équipe de J.Y. Reginster cherche aussi à en préciser le mécanisme, avec comme objectif de proposer des traitements substitutifs, visant à enrayer la perte de la masse osseuse liée à cette maladie.

\section{L'immunologie}

L'immunologie est proche de l'endocrinologie car elle fait appel aussi à des communications intercellulaires relayées par des molécules exportées, en l'occurrence les cytokines.

On trouve une activité de recherche importante en rapport avec l'immunologie antitumorale, déjà citée. Par ailleurs, M. Moutschen et al. étudient un modèle murin d'immunodéficience acquise induite par un rétrovirus (MAIDS), dans lequel ils cherchent à déterminer quels sont les chaînons des signaux d'activation cellulaire qui sont altérés dans cette affection. Le groupe du laboratoire de référence SIDA, dirigé par D. Sondag-Thull collabore à de nombreux programmes d'études sur les corécepteurs et sur la résistance médicamenteuse chez les patients infectés par le VIH.

Insistons aussi sur l'importante activité de transplantation d'organes (cœur, reins, poumon, pancréas, foie) réalisée par les équipes chirurgicales de M. Meurisse, P. Honoré, N. Jacquet, R. Limet au CHU. Cela entraîne une activité intensive des laboratoires d'immunologie clinique, de microbiologie et de virologie, mais aussi des programmes de recherche importants. Ceux-ci se sont développés récemment dans deux directions qui visent à élargir les possibilités de transplantation. L'un, mené par J.O. Defraigne au Laboratoire de Chirurgie expérimentale et au Centre de l'Oxygène dirigé par M. Lamy, vise à mettre au point des méthodes optimales de conservation des organes à transplanter, et ce, en particulier, en cherchant à limiter les effets cytotoxiques de l'ischémie-reperfusion, via la formation de radicaux libres et de métabolites de l'oxygène. L'autre voie, menée par un groupe présidé par Alexandre et installé en collaboration avec plusieurs équipes de la Faculté de Médecine vétérinaire, est celle de la xénotransplantation, l'animal choisi étant le porc.

\section{L'immunopathologie}

En immunopathologie, on doit aussi citer les élégants travaux de G. Pierard, P. Paquet et al. sur l'identification des cellules inflammatoires et des cytokines impliquées dans des maladies cutanées comme le syndrome de Lyell et la réaction du greffon contre l'hôte. Le TNF- $\alpha$ y joue un rôle fort important. Évoquons aussi les recherches de M. Mahieu et M. Malaise sur les modifications induites par le méthotrexate dans les lésions immuno-induites de la polyarthrite rhumatoïde.

Au niveau très fondamental, E. Heinen et son équipe s'intéressent au rôle des cellules folliculaires dendritiques, qu'ils ont été parmi les premiers à isoler, dans les réactions immunitaires, tandis que M.P. Defresne, Ch. Humblet, V. Geenen et leurs collègues cherchent à comprendre le rôle du thymus dans le sytème immunitaire.

\section{La cardiologie}

Un autre point fort de l'Hôpital universitaire est le centre de Cardiologie et de Chirurgie cardiovasculaire; cela explique le développement de programme de recherches dans ces domaines, dont certains ont une orientation très proche de la clinique, par exemple dans le cadre de l'électrophysiologie et de l'échocar- diographie, de l'imagerie et de la biologie clinique (H. Kulbertus, D. Soyeur, L. Pierard, V. Legrand, J.P. Chapelle).

Par ailleurs, il convient de mentionner les travaux de M. Radermecker sur la cardiomyoplastie, et donc sur l'utilisation d'un muscle squelettique en chirurgie cardio-aortique, et sur les anévrysmes aortiques, où $\mathrm{R}$. Limet, C. Lapiere, B. Nusgens et N. Sakalihassan décrivent des altérations biochimiques qui servent de marqueurs du risque de rupture.

\section{La gynécologie-obstétrique}

Le département de gynécologie-obstétrique présidé par J.M. Foidart est centré, comme signalé plus haut à l'hôpital de la Citadelle, mais dispose d'antennes au Sart Tilman et dans deux autres hôpitaux liégeois.

Son activité engendre de nombreuses recherches à orientation clinique sur la maîtrise de la fécondité (A. Demoulin), sur l'échographie fotale (J.P. Schaaps), sur les tests prédictifs (entre autres génétiques), prénataux (L. Koulischer, A. Verloes), sur les facteurs de risque de la prématurité $(\mathrm{H}$. Thoumsin) et enfin sur toutes les affections liées à la prématurité (J. Rigo). Ces dernières études étaient menées par le service de Néonatologie attaché au Département de Pédiatrie. Par ailleurs, J.M. Foidart et ses collaborateurs, très impliqués dans la recherche cancérologique étudient les mécanismes de l'implantation trophoblastique, et mettent en exergue le parallélisme entre cette implantation et l'invasion stromale par les cellules cancéreuses en particulier à travers le rôle des métalloprotéinases. Ces enzymes sont au centre de nombreuses recherches dans notre Faculté, d'ailleurs en rapport avec le rôle du tissu conjonctif dans les phénomènes physiologiques ou pathologiques.

\section{Le laboratoire de biologie des tissus conjonctifs}

Le laboratoire de biologie des tissus conjonctifs, créé par C. Lapiere et dirigé aujourd'hui par B. NusgensRichelle, outre sa collaboration aux recherches déjà mentionnées, a réussi le clonage de l'amino-colla-
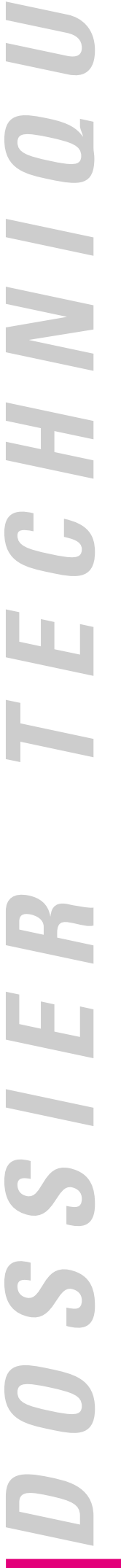

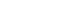


gène peptidase; il s'intéresse aussi aux effets d'influences physiques (forces mécaniques, hypogravité, champs électromagnétiques) sur les fibroblastes du tissu conjonctif et aux greffes de kératinocytes, en collaboration avec M. de la Brassine du service de Dermatologie. Il collabore aussi avec les services de l'Institut de Dentisterie, qui cherchent à étudier les conditions de régénération osseuse, indispensable au développement de la chirurgie orale. Les chirurgiens orthopédistes fréquentent aussi ce laboratoire pour y analyser les séquelles des poses de prothèse articulaire et le devenir de greffons osseux au niveau du rachis. Enfin, M. Malaise et ses collaborateurs analysent aussi les métalloprotéinases en pathologie ostéo-articulaire.

\section{La gastroentérologie}

Les recherches en gastroentérologie sont menées sur le plan fondamental, par G. Dandrifosse et al., qui s'efforcent d'analyser les mécanismes exogènes, telles la présence de polyamines dans le lait, intervenant dans le développement des allergies alimentaires. J. Belaiche, E. Louis et D. Franchimont s'intéressent aux maladies inflammatoires de l'intestin; ils ont montré l'implication de zones situées sur les chromosomes 3, 7 et 12 dans le déterminisme de ces maladies; le gène du TNF pourrait être important pour la modulation de la maladie; ils étudient aussi des cytokines comme IL-6, IL-2 et IL-10 comme marqueurs d'évolutivité.

\section{L'École de Santé publique}

L'École de Santé publique, grâce à A. Albert, P. Mairiaux et J.Y. Reginster, mène des recherches originales sur l'épidémiologie et sur la promotion de la santé.

\section{L'Institut de pharmacie}

L'Institut de pharmacie, déjà mentionné, s'installe dès la fin de 1997 , sur le site de l'Hôpital universitaire. Ses chercheurs se rapprochant ainsi des médecins trouveront dans cette ments pour développer encore leurs recherches.

J. Delarge, B. Masereel, B. Pirotte et J.F. Liégeois sont à la recherche de nouveaux produits de synthèse destinés à l'industrie pharmaceutique. Ce groupe recherche aujourd'hui des analogues de l'amiloride, un inhibiteur des canaux potassiques; ils étudient aussi des modulateurs de ces canaux; ils développent des molécules hétérocycliques - les coumarines - qui sont des inhibiteurs de protéases à sérine ; à partir du torasémide, un diurétique qu'ils ont mis au point il y a quelques années, qui s'est révélé en outre un inhibiteur du thromboxane, ils synthétisent de nouvelles molécules inhibitrices de ce thromboxane. Ces chercheurs étudient aussi certains psychotropes.

C'est à partir de produits naturels et en particulier de plantes, que L. Angenot et son équipe cherchent à isoler de nouvelles molécules à intérêt pharmaceutique, en particulier pour la cancérologie ou contre l'infestation par protozoaires.

Dans le même institut, certaines recherches sont «analytiques». Ainsi, L. Thunus et ses collègues développent des immunodosages homogènes de médicaments (stéroïdes, antibiotiques). J. Crommen, J. Bonnard, P. Hubert et leurs collaborateurs sont spécialisés dans les méthodes de chromatographie (HPLC, GC, CE...) pour le dosage de médicaments.

Enfin L. Delattre, dans son service de technologie pharmaceutique, travaille sur les formes galéniques les plus appropriées pour l'efficacité médicamenteuse. Citons l'inclusion de principes actifs de nature peptidique dans des nanoparticules, le développement de nouvelles formes topiques, à base de coévaporés dithranol-polyvinylpynolidone, pour le traitement du psoriasis, l'utilisation de cyclodextrines pour augmenter la solubilité aqueuse de principes actifs.

\section{Les fondations} de recherche liées à la Faculté de Médecine

Comme chacun le sait (malheureusement !), la recherche biomédicale est insuffisamment soutenue en Belgique par les pouvoirs publics. Les subsides proviennent soit du gouvernement fédéral, soit de la Région wallonne (pour ce qui concerne la recherche appliquée), soit de la Communauté française. Cette dernière finance la recherche à travers le budget alloué à l'Université (mais la part de celui-ci qu'elle peut réellement consacrer à la recherche est faible) et à travers le Fonds national de la Recherche scientifique. Celui-ci s'appuie sur l'opération Télévie, organisée par un média télévisuel, pour financer la recherche cancérologique.

A ces fonds publics, s'ajoutent des sources de fondations privées ou d'associations privées ou encore de certaines entreprises bancaires.

Plusieurs groupes sont intégrés dans des projets européens, relevant des programmes Biotech, Biomed ou Fusion nucléaire. Mais les moyens restent insuffisants.

La volonté de la Faculté de Médecine de développer une politique de recherche dynamique et à attirer ses jeunes diplômés l'a amenée à créer deux fondations destinées à apporter des soutiens financiers complémentaires aux chercheurs sous forme de bourses de doctorats ou de voyage ou de crédits de fonctionnement ou d'équipement. La plus ancienne de ces fondations est le Centre anticancéreux près l'Université de Liège, qui obtient ses fonds de legs et de dons privés. Outre les aides susmentionnées, il finance partiellement l'Animalerie de la Faculté de Médecine, la Bibliothèque de la Faculté de Médecine et récemment il vient de financer l'installation d'un laboratoire P3 pour la thérapie génique expérimentale. Le Centre consacre annuellement 10 à 15 millions de francs belges à la recherche. La Fondation Léon Fredericq est plus jeune. Elle aide tous les domaines de la recherche biomédicale et obtient ses fonds de dons privés ou d'entreprises, de legs ou par des manifestations culturelles.

Enfin, l'Hôpital universitaire ne reste pas indifférent; dès la fin des années 1980, Paul Franchimont avait créé le Fonds de la Recherche Facultaire, à partir de prélèvements effectués sur les honoraires médicaux. Ce fonds permet aujourd'hui de rémunérer une vingtaine de techniciens de recherche dans les laboratoires de 
la Faculté. Plus récemment, le Conseil médical de l'Hôpital a décidé de créer des mandats de formation en recherche clinique pour ses jeunes médecins; une dizaine de tels mandats ont été attribués pour l'année 1997-1998; en outre, il consacre une vingtaine de millions de francs belges au financement de projets de recherche à visée clinique. En d'autres termes, l'hôpital investit environ 70 millions de francs belges annuellement dans la recherche biomédicale, c'est une situation quasi unique en Belgique.

Cela indique combien, malgré les mauvaises conditions de financement par l'Etat, la Faculté de Médecine de l'Université de Liège et son hôpital entendent promouvoir la recherche

\section{Summary}

Biomedical research in the Faculty of Medecine at the University of Liège

The Faculty of Medecine at the University of Liège is closely linked to the University Hospital. The Biomedical Research is very active in many fields, particularly in experimental and clinical oncology, neurosciences and endocrinology. Local Foundations (the Leon Fredericq Foundation and the Anticancer Centre) have been developed to support research in the Faculty.

\section{J. Boniver}

Professeur d'anatomie pathologique, Doyen de la faculté de médecine. Université de Liège, Docanat de la faculté de médecine, Tour de pathologie, B35, Sart Tilman, B-4000 Liège, Belgique.
TIRÉS À PART J. Boniver.

\title{
Vle Journée ANNUElLE du Club des NeUROSCIENCES de CréteIL
}

Sous l'égide de l'Université Paris XII-Val-de-Marne et de l'Institut Mondor de Médecine Moléculaire (IM3) avec le soutien de l'ANPP

\section{Le Jeudi 28 mai 1998}

\author{
Faculté de Médecine de Créteil - Amphi 2
}

\section{GLIE - GLIOSE - GLIOME}

9 h 00 Introduction

Guy Atlan - Président de l'Institut Mondor de Médecine Moléculaire (IM3)

Modérateurs : Brigitte Onténiente, Pierre Cesaro

9 h 15 - 10 h 15 Jacques Glowinski (Inserm U. 114 /Collège de France, Paris)

Relations astrocyto-neuronales. Rôle de l'acide arachidonique

10 h 30 - 11 h 30 Michel Mallat (Inserm U.495, Pitié-Salpêtrière, Paris)

Croissance et réaction microgliale : mécanisme et aspect fonctionnel

11 h 30 - 12 h 30 Dionysia Theodosis (Inserm U.378, Bordeaux)

Plasticité des interactions neurone/glie

Modérateurs : Josette Cadusseau, Romain Ghérardi

14 h 00 - 15 h 00 Manuel Nieto-Sampedro (Institut Cajal - Madrid)

Inhibitions of neurite outgrowth in brain gliotic tissue

15 h 00 - 16 h 00 Anne Baron-Van Evercooren (Inserm CJF 96-08, Pitié-Salpêtrière, Paris)

Approches expérimentales de la remyélinisation du SNC

16 h 00 - 17 h 00 Catherine Daumas-Duport (Service d'Anatomie Pathologique, St Anne, Paris)

Gliomes. Étude anatomo-pathologique

Club des Neurosciences de Créteil, Inserm U.421, 8, rue du Général-Sarrail, 94010 Créteil Cedex, France Téléphone : 0149813682 - Télécopie : 0149813709 -E-mail : ribeil@im3.inserm.fr 